PROCEEDINGS OF THE

AMERICAN MATHEMATICAL SOCIETY

Volume 138, Number 6, June 2010, Pages 2235-2244

S 0002-9939(10)10240-8

Article electronically published on February 12, 2010

\title{
ASSOUAD-NAGATA DIMENSION OF NILPOTENT GROUPS WITH ARBITRARY LEFT INVARIANT METRICS
}

\author{
J. HIGES
}

(Communicated by Alexander N. Dranishnikov)

\begin{abstract}
Suppose $G$ is a countable, not necessarily finitely generated, group. We show $G$ admits a proper, left invariant metric $d_{G}$ such that the AssouadNagata dimension of $\left(G, d_{G}\right)$ is infinite, provided the center of $G$ is not locally finite. As a corollary we solve two problems of A. Dranishnikov.
\end{abstract}

\section{INTRODUCTION}

The asymptotic dimension was introduced by Gromov in [16] as a coarse invariant to study the geometric structure of finitely generated groups. Asymptotic dimension has been studied in many classes of groups such as nilpotent groups, amalgamations, and relative hyperbolic groups (see for example [2, 3], 4, 21, [22] and [25]). We refer to [5] for a survey about this topic. Closely related to the asymptotic dimension is the asymptotic dimension of linear type. It is called asymptotic Assouad-Nagata dimension in honor of Patrice Assouad, who introduced it in [1, inspired by the ideas of Nagata. That dimension can be considered as the linear version of the asymptotic dimension. In recent years a part of the research activity was focused on this dimension and its relationship with the asymptotic dimension (see for example [19], 12], 13, 6], 7], 9], 8], 20, [15, 18]). One of the main problems of interest consists of studying the differences between the asymptotic dimension and the asymptotic Assouad-Nagata dimension in the context of the geometric group theory. In particular there are two main questions:

(1) Given a finitely generated group $G$ with a word metric $d_{G}$, are the asymptotic dimension and the asymptotic Assouad-Nagata dimension of $\left(G, d_{G}\right)$ equal?

(2) In the case the two dimensions differ, must their difference be infinite?

It is known that the first question has an affirmative answer for abelian groups, finitely presented groups of asymptotic dimension one, and hyperbolic groups. But in general the answer is negative. Nowak (20) found for every $n \geq 1$ a finitely generated group of asymptotic dimension $n$ but of infinite asymptotic AssouadNagata dimension.

Received by the editors June 11, 2009, and, in revised form, October 3, 2009.

2000 Mathematics Subject Classification. Primary 54F45; Secondary 55M10, 54C65.

Key words and phrases. Assouad-Nagata dimension, asymptotic dimension, nilpotent groups.

The author is supported by Grant AP2004-2494 from the Ministerio de Educación y Ciencia, Spain, and project MEC, MTM2006-0825. He also thanks Jerzy Dydak and N. Brodskyi for helpful comments and support.

(C)2010 American Mathematical Society Reverts to public domain 28 years from publication 
As far as the author knows the second question is still open. There is no example of a finitely generated group such that the asymptotic dimension is strictly smaller than the asymptotic Assouad-Nagata dimension but both are finite. In [18 the second question was solved in a more general context. It was proved that for every $n$ and $m$ there exists a countable abelian group (nonfinitely generated) with a proper left invariant metric such that the group is of asymptotic dimension $n$ but of asymptotic Assouad-Nagata dimension equal to $n+m$. Proper left invariant metrics are natural generalizations of word metrics. Therefore it is natural to ask the same two problems in the case of finitely generated groups equipped with proper left invariant metrics.

The aim of this paper is to study the behavior of the asymptotic Assouad-Nagata dimension in nilpotent groups with proper left invariant metrics. It is highly likely that both dimensions coincide in nilpotent groups equipped with word metrics (see [10]). For example in [15] their coincidence was proved for the Heisenberg group. We will show that every nilpotent group admits a proper left invariant metric such that both dimensions are different. Our result arose from attempting to solve the following two questions of Dranishnikov [10]:

Problem 1.1 (Dranishnikov [10]). Does $\operatorname{dim}_{A N}(\mathbb{Z}, d)=1$ for every left invariant metric on $\mathbb{Z}$ ?

Problem 1.2 (Dranishnikov [10]). Does $\operatorname{dim}_{A N}(\Gamma \times \mathbb{Z})=\operatorname{dim}_{A N}(\Gamma)+1$ for any left invariant metric on $\mathbb{Z}$ ?

Notice that in $\left[12\right.$, Dranishnikov and Smith proved that $\operatorname{asdim}_{A N}(G \times \mathbb{Z})=$ $\operatorname{asdim}_{A N}(G)+1$ for every finitely generated group $G$, but in this case the metrics considered were the word metrics.

We recall that a group is locally finite if all of its finitely generated subgroups are finite. Our main theorem deals with a larger class of groups than that of nilpotent groups:

Theorem 1.3. If the center of a countable group $G$ is not locally finite, then there exists a proper left invariant metric $d_{G}$ on $G$ satisfying $\operatorname{asdim}_{A N}\left(G, d_{G}\right)=\infty$.

By applying the above theorem to $G=\mathbb{Z}$, the two questions of Dranishnikov are solved in the negative.

The key ingredient of the proof was introduced by the author in 18. In that paper it was shown that if there exists a sequence of isometric embeddings (up to dilatation) of balls $\left\{B\left(0, k_{i}\right)\right\}_{i \in \mathbb{N}}$ of $\mathbb{Z}^{m}$ into a metric space $X$ where the sequence of radii $k_{i}$ tends to infinity, then the asymptotic Assouad-Nagata dimension of $X$ is greater than $m$. That result should be viewed as applying the philosophy of Whyte [26] in a rather restricted form. Instead of looking for subsets of a group $G$ that are bi-Lipschitz equivalent to $\mathbb{Z}^{n}$, we are constructing groups that contain re-scaled copies of large balls in $\mathbb{Z}^{n}$.

Section 3 is devoted to a key ingredient used in Section 4 to present proofs of the main results.

\section{Preliminaries}

Let $s$ be a positive real number. An $s$-scale chain (or $s$-path) between two points $x$ and $y$ of a metric space $\left(X, d_{X}\right)$ is defined as a finite sequence of points $\left\{x=x_{0}, x_{1}, \ldots, x_{m}=y\right\}$ such that $d_{X}\left(x_{i}, x_{i+1}\right)<s$ for every $i=0, \ldots, m-1$. A 
subset $S$ of a metric space $\left(X, d_{X}\right)$ is said to be $s$-scale connected if there exists an $s$-scale chain contained in $S$ for every two elements of $S$.

Definition 2.1. A metric space $\left(X, d_{X}\right)$ is said to be of asymptotic dimension at most $n$ (notation asdim $(X, d) \leq n$ ) if there is an increasing function $D_{X}: \mathbb{R}_{+} \rightarrow \mathbb{R}_{+}$ such that for all $s>0$ there is a cover $\mathcal{U}=\left\{\mathcal{U}_{0}, \ldots, \mathcal{U}_{n}\right\}$ so that the $s$-scale connected components of each $\mathcal{U}_{i}$ are $D_{X}(s)$-bounded; i.e. the diameter of such components is bounded by $D_{X}(s)$.

The function $D_{X}$ is called an $n$-dimensional control function for $X$. Depending on the type of $D_{X}$ one can define the following two invariants:

A metric space $\left(X, d_{X}\right)$ is said to be of Assouad-Nagata dimension at most $n$ (notation $\operatorname{dim}_{A N}(X, d) \leq n$ ) if it has an $n$-dimensional control function $D_{X}$ of the form $D_{X}(s)=C \cdot s$ with $C>0$ some fixed constant.

A metric space $\left(X, d_{X}\right)$ is said to be of asymptotic Assouad-Nagata dimension at most $n$ (notation $\operatorname{asdim}_{A N}(X, d) \leq n$ ) if it has an $n$-dimensional control function $D_{X}$ of the form $D_{X}(s)=C \cdot s+k$ with $C>0$ and $k \in \mathbb{R}$ two fixed constants.

It is clear from the definition that for every metric space $\left(X, d_{X}\right), \operatorname{asdim}\left(X, d_{X}\right) \leq$ $\operatorname{asdim}_{A N}\left(X, d_{X}\right)$.

One important fact about the asymptotic dimension is that it is invariant under coarse equivalences. Given a map $f:\left(X, d_{X}\right) \rightarrow\left(Y, d_{Y}\right)$ between two metric spaces it is said to be a coarse embedding if there exist two increasing functions $\rho_{+}: \mathbb{R}_{+} \rightarrow \mathbb{R}_{+}$and $\rho_{-}: \mathbb{R}_{+} \rightarrow \mathbb{R}_{+}$with $\lim _{x \rightarrow \infty} \rho_{-}(x)=\infty$ such that

$$
\rho_{-}\left(d_{X}(x, y)\right) \leq d_{Y}(f(x), f(y)) \leq \rho_{+}\left(d_{X}(x, y)\right) \text { for every } x, y \in X .
$$

Now a coarse equivalence between two metric spaces $\left(X, d_{X}\right)$ and $\left(Y, d_{Y}\right)$ is defined as a coarse embedding $f:\left(X, d_{X}\right) \rightarrow\left(Y, d_{Y}\right)$ for which there exists a constant $K>0$ such that $d_{Y}(y, f(X)) \leq K$ for every $y \in Y$. If there exists a coarse equivalence between $X$ and $Y$, both spaces are said to be coarsely equivalent.

The metric spaces in which we are interested are countable groups with proper left invariant metrics.

Definition 2.2. A metric $d_{G}$ defined in a group $G$ is said to be a proper left invariant metric if it satisfies the following conditions:

(1) $d_{G}\left(g_{1} \cdot g_{2}, g_{1} \cdot g_{3}\right)=d_{G}\left(g_{2}, g_{3}\right)$ for every $g_{1}, g_{2}, g_{3} \in G$.

(2) For every $K>0$ the number of elements $g$ of $G$ such that $d\left(1_{G}, g\right)_{G} \leq K$ is finite.

The following basic result of Smith (see also [23]) will be used to get the main theorem.

Theorem 2.3. Two proper left invariant metrics defined in a countable group are coarsely equivalent.

Associated to each proper left invariant metric there exists a proper norm.

Definition 2.4. A map $\|\cdot\|_{G}: G \rightarrow \mathbb{R}_{+}$is called a proper norm if it satisfies the following conditions:

(1) $\|g\|_{G}=0$ if and only if $g=1_{G}$.

(2) $\|g\|_{G}=\left\|g^{-1}\right\|_{G}$ for every $g \in G$.

(3) $\|g \cdot h\|_{G} \leq\|g\|_{G}+\|h\|_{G}$ for every $g, h \in G$.

(4) For every $K>0$ the number of elements of $G$ such that $\|g\|_{G} \leq K$ is finite. 
It is clear that there is a one-to-one correspondence between proper norms and proper left invariant metrics.

We are interested in methods to get proper norms with some special properties. In general this task is not easy. In this paper we will use the method of weights described by Smith in 24. Let $S$ be a symmetric system of generators (possibly infinite) of a countable group $G$, and let $\omega: S \rightarrow \mathbb{R}_{+}$be a function (weight function or system of weights) that satisfies:

(1) $\omega(s)=0$ if and only if $s=1_{G}$.

(2) $\omega(s)=w\left(s^{-1}\right)$.

(3) $\omega^{-1}[0, N]$ is finite for every $N$.

The function $\|\cdot\|_{w}: G \rightarrow \mathbb{R}_{+}$defined by

$$
\|g\|_{w}=\min \left\{\sum_{i=1}^{n} w\left(s_{i}\right) \mid g=\Pi_{i=1}^{n} s_{i}, s_{i} \in S\right\}
$$

is a proper norm. Such a norm will be called the proper norm generated by the system of weights $\omega$ and the associated left invariant metric will be the left invariant metric generated by the system of weights $\omega$.

Remark 2.5. (1) If we define $w(g)=1$ for all the elements $g \in S$ of a finite generating system $S \subset G$ ( $G$ a finitely generated group), we will obtain the usual word metric.

(2) Notice that if we have a proper norm $\|\cdot\|_{G}$ in a countable group $G$ and we take the system of weights defined by $\omega(g)=\|g\|_{G}$, then the proper norm $\|\cdot\|_{\omega}$ generated by this system of weights coincides with $\|\cdot\|_{G}$.

(3) We can easily construct integer valued proper left invariant metrics by choosing weight functions with integer ranges.

\section{LOWER BOUndS For Assouad-NAGATA DimEnsion}

The aim of this section is to give a sufficient condition on a metric space $\left(X, d_{X}\right)$ that implies $\operatorname{asdim}_{A N}\left(X, d_{X}\right) \geq n$ for some $n$. To get that condition we will use the notion of an asymptotic cone.

Let $\left(X, d_{X}\right)$ be a metric space. Given a non-principal ultrafilter $\omega$ of $\mathbb{N}$ and a sequence $\left\{x_{n}\right\}_{n \in \mathbb{N}}$ of points of $X$, the $\omega$-limit of $\left\{x_{n}\right\}_{n \in \mathbb{N}}$ (notation: $\lim x_{n}=y$ ) is an element $y$ of $X$ such that for every neighborhood $U_{y}$ of $y$ the set $F_{U_{y}}{ }^{\omega}=\left\{n \mid x_{n} \in U_{y}\right\}$ belongs to $\omega$. It can be proved easily that the $\omega$-limit of a sequence always exists in a compact space.

Assume $\omega$ is a non-principal ultrafilter of $\mathbb{N}$. Let $d=\left\{d_{n}\right\}_{n \in \mathbb{N}}$ be an $\omega$-divergent sequence of positive real numbers and let $c=\left\{c_{n}\right\}_{n \in \mathbb{N}}$ be any sequence of elements of $X$. Now we can construct the asymptotic cone (notation: $C_{0} e_{\omega}(X, c, d)$ ) of $X$ as follows:

First consider the set of all sequences $\left\{x_{n}\right\}_{n \in \mathbb{N}}$ of elements of $X$ such that the sequence $\left\{\frac{d_{X}\left(x_{n}, c_{n}\right)}{d_{n}}\right\}_{n \in \mathbb{N}}$ is bounded. In that set take the pseudo metric given by

$$
D\left(\left\{x_{n}\right\}_{n \in \mathbb{N}},\left\{y_{n}\right\}_{n \in \mathbb{N}}\right)=\lim _{\omega} \frac{d_{X}\left(x_{n}, y_{n}\right)}{d_{n}} .
$$

By identifying sequences whose distances are 0 we get the metric space $\operatorname{Cone}_{\omega}(X, c, d)$. 
Asymptotic cones were originally introduced by Gromov in [16] using GromovHausdorff convegence. There has been a lot of research relating properties of groups with topological properties of its asymptotic cones. For example a finitely generated group is virtually nilpotent if and only if all its asymptotic cones are locally compact [17. or a group is hyperbolic if and only if all of its asymptotic cones are $\mathbb{R}$-trees ([16] and [14).

In [15] the authors show the following relationship between the topological dimension of an asymptotic cone and the asymptotic Assouad-Nagata dimension of the space:

Theorem 3.1 (Dydak, Higes [15]).

$$
\operatorname{dim}\left(\text { Cone }_{\omega}(X, c, d) \leq \operatorname{dim}_{A N}\left(\text { Cone }_{\omega}(X, c, d) \leq \operatorname{asdim}_{A N}\left(X, d_{X}\right)\right.\right.
$$

for any metric space $\left(X, d_{X}\right)$ and every asymptotic cone Cone $e_{\omega}(X, c, d)$.

We now recall the following:

Definition 3.2. A function $f:\left(X, d_{X}\right) \rightarrow\left(Y, d_{Y}\right)$ between metric spaces is said to be a dilatation if there exists a constant $C \geq 1$ such that $d_{Y}(f(x), f(y))=$ $C \cdot d_{X}(x, y)$ for every $x, y \in X$.

The number $C$ will be called the dilatation constant.

In the following proposition dilatations will be from balls of $\mathbb{Z}^{n}$ with the $l_{1}$-metric to general metric spaces.

Proposition 3.3. Let $\left(X, d_{X}\right)$ be a metric space and let $\left\{k_{m}\right\}_{m \in \mathbb{N}}$ be an increasing sequence of natural numbers. If for some $n \in \mathbb{N}$ there is a sequence of dilatations $\left\{f_{m}\right\}_{m=1}^{\infty}$ of the form $f_{m}: B^{n}\left(0, k_{m}\right) \rightarrow\left(X, d_{X}\right)$ with $B^{n}\left(0, k_{m}\right) \subset \mathbb{Z}^{n}$ the ball of radius $k_{m}$, then there exists an asymptotic cone Cone $\omega_{\omega}(X, c, d)$ of $\left(X, d_{X}\right)$ such that $[-1,1]^{n} \subset$ Cone $_{\omega}(X, c, d)$.

Proof. Suppose $\left(X, d_{X}\right)$ and $\left\{k_{m}\right\}_{m \in \mathbb{N}}$ are as in the hypothesis. Let us first prove the case $n=1$. Assume that $\left\{C_{m}\right\}_{m \in \mathbb{N}}$ is the sequence of dilatation constants of $\left\{f_{m}\right\}_{m \in \mathbb{N}}$. Take $\omega$ to be some ultrafilter of $\mathbb{N}$ and define $c=\left\{f_{m}(0)\right\}_{m=1}^{\infty}$ and $d=\left\{d_{m}\right\}_{m=1}^{\infty}$ with $d_{m}=C_{m} \cdot k_{m}$. We will prove that $C_{0 n e}(X, c, d)$ contains $[-1,1]$. For each $t \in[-1,1]$ let $A_{m}^{t}$ be the subset $\left\{-k_{m}, \ldots, k_{m}\right\}$ such that $x \in A_{m}^{t}$ if and only if the distance between $\frac{C_{m} \cdot x}{d_{m}}$ and $t$ is minimum. Notice that this implies that the distance between $C_{m} \cdot x$ and $d_{m} \cdot t$ is less than $C_{m}$. Take now the sequence $\left\{r_{m}^{t}\right\}_{m=1}^{\infty}$ where $r_{m}^{t}$ is the infimum of $A_{m}^{t}$.

Define the map $g:[-1,1] \rightarrow$ Cone $_{\omega}(X, c, d)$ by $g(t)=x^{t}$ if the sequence $\left\{f_{m}\left(r_{m}^{t}\right)\right\}_{m=1}^{\infty}$ is in the class $x^{t}$. As

$$
\lim _{\omega} \frac{d\left(f_{m}(0), f_{m}\left(r_{m}^{t}\right)\right)}{d_{m}}=\lim _{\omega} \frac{C_{m} \cdot\left|r_{m}^{t}\right|}{d_{m}} \leq \lim _{\omega} \frac{C_{m} \cdot k_{m}}{d_{m}}=1,
$$

the map is well defined. Let us prove it is an isometry. From the definition of $r_{m}^{t}$ we get that if $t_{1}<t_{2}$, then $r_{m}^{t_{1}} \leq r_{m}^{t_{2}}$, which implies $\lim _{\omega} \frac{d\left(f_{m}\left(r_{m}^{t_{1}}\right), f_{m}\left(r_{m}^{t_{2}}\right)\right)}{d_{m}}=$ $\lim _{\omega} \frac{C_{m}\left(r_{m}^{t_{2}}-r_{m}^{t_{1}}\right)}{d_{m}}$. So the unique thing we need to show is that $\lim _{\omega} \frac{C_{m} \cdot r_{m}^{t}}{d_{m}}=t$ for every $t$. Notice that we have $\lim _{\omega} \frac{C_{m}}{d_{m}}=0$ as $\lim _{\omega} k_{m}=\infty$ but $\lim _{\omega} \frac{{ }^{\omega} C_{m} \cdot k_{m}}{d_{m}}=1$. This implies that given $\epsilon>0$ there exists $G_{\epsilon} \in \omega$ such that $\frac{C_{m}}{d_{m}}<\epsilon$ for every $m \in G_{\epsilon}$. Therefore by the choice of $r_{m}^{t}$, if $m \in G_{\epsilon}$ we have $\left|C_{m} \cdot r_{m}^{t}-d_{m} \cdot t\right|<C_{m}$ and then $\left|\frac{C_{m} \cdot r_{m}^{t}}{d_{m}}-t\right|<\frac{C_{m}}{d_{m}} \leq \epsilon$. 
Now let us do the general case. Let $\left(s_{1}, \ldots, s_{n}\right) \in[-1,1]^{n}$. By the previous case we get that for every $j=1, \ldots, n$ there exists a sequence $\left\{r_{m}^{s_{j}}\right\}_{m \in \mathbb{N}}$ with $r_{m}^{s_{j}} \in$ $\left\{-k_{m}, \ldots, k_{m}\right\}$ such that $\lim _{\omega} \frac{C_{m} \cdot r_{m}^{t}}{d_{m}}=s_{j}$. In a similar way as before, we construct a map $g:[-1,1]^{n} \rightarrow$ Cone $_{\omega}(X, c, d)$ by defining $g\left(s_{1}, \ldots, s_{m}\right)$ as the class that contains the sequence $\left\{f_{m}\left(r_{m}^{s_{1}}, \ldots, r_{m}^{s_{n}}\right)\right\}_{m=1}^{\infty}$. To finish the proof it will be enough to check that for every $s, t \in[-1,1]^{n}$ with $s=\left(s_{1}, \ldots, s_{n}\right)$ and $t=\left(t_{1}, \ldots, t_{n}\right)$, the following equality holds:

$$
\lim _{\omega} \frac{d_{X}\left(f_{m}\left(r_{m}^{s_{1}}, \ldots, r_{m}^{s_{n}}\right), f_{m}\left(r_{m}^{t_{1}}, \ldots, r_{m}^{t_{n}}\right)\right)}{d_{m}}=\sum_{i=1}^{n}\left|s_{i}-t_{i}\right| .
$$

As $f_{m}$ is a dilatation of constant $C_{m}$ we can write:

$$
\lim _{\omega} \frac{d_{X}\left(f_{m}\left(r_{m}^{s_{1}}, \ldots, r_{m}^{s_{n}}\right), f_{m}\left(r_{m}^{t_{1}}, \ldots, r_{m}^{t_{n}}\right)\right)}{d_{m}}=\sum_{i=1}^{n} \lim _{\omega} \frac{C_{m} \cdot\left|r_{m}^{s_{i}}-r_{m}^{t_{i}}\right|}{d_{m}}
$$

And again by the case $n=1$ we can deduce that the last term satisfies the equality

$$
\sum_{i=1}^{n} \lim _{\omega} \frac{C_{m} \cdot\left|r_{m}^{s_{i}}-r_{m}^{t_{i}}\right|}{d_{m}}=\sum_{i=1}^{n}\left|s_{i}-t_{i}\right| .
$$

From the previous proposition we can get the following result, which is one of the ingredients of the main theorem.

Corollary 3.4. If for metric space $\left(X, d_{X}\right)$ and for some $n \in \mathbb{N}$ there exists a sequence of dilatations $f_{m}: B^{n}\left(0, k_{m}\right) \rightarrow\left(X, d_{X}\right)$ with $\lim _{m \rightarrow \infty} k_{m}=\infty$ and $B^{n}\left(0, k_{m}\right) \subset \mathbb{Z}^{n}$ the ball of radius $k_{m}$, then $\operatorname{asdim}_{A N}\left(X, d_{X}\right) \geq n$.

Proof. By Proposition 3.3 we get that there exists an asymptotic cone of $X$ such that $[-1,1]^{n} \subset$ Cone $_{\omega}(X, c, d)$. Applying Theorem 3.1 we obtain immediately

$$
n \leq \operatorname{dim}\left(\text { Cone }_{\omega}(X, c, d)\right) \leq \operatorname{dim}_{A N}\left(\text { Cone }_{\omega}(X, c, d)\right) \leq \operatorname{asdim}_{A N}\left(X, d_{X}\right) .
$$

\section{MAin Results}

The idea of the proof of the main theorem consists in creating a metric inductively. In each step we will construct a new metric that satisfies two conditions. The first condition says that the new metric does not change a sufficiently large ball of the old metric. The second condition implies there is a dilatation from some sufficiently large ball of $\mathbb{Z}^{n}$ into the group with the new metric. In fact that dilatation will be the restriction of some homomorphism $f: \mathbb{Z}^{n} \rightarrow G$. Then we will apply Corollary 3.4. The following lemma could be considered as the induction step.

Lemma 4.1. Let $G$ be a finitely generated group such that its center is not locally finite. Let $\|\cdot\|_{G}$ be a proper norm. In such conditions for every $k, m, R \in \mathbb{N}$ there exists a proper norm $\|\cdot\|_{\omega}$ that satisfies the following conditions:

(1) $\|g\|_{\omega} \leq\|g\|_{G}$.

(2) $\|g\|_{G}=\|g\|_{\omega}$ if $\|g\|_{\omega} \leq R$.

(3) There is a homomorphism $f: \mathbb{Z}^{m} \rightarrow G$ such that the restriction $\left.f\right|_{B(0, k)}$ of $f$ to the ball radius $k$ is a dilatation in $\left(G, d_{\omega}\right)$. 
Proof. Suppose $k, m$ and $R$ are given and let $a$ and $C$ be two natural numbers that satisfy

$$
R<C<\frac{a}{2 \cdot k \cdot m^{2}} .
$$

As the center of $G$ is not locally finite there exists an element $g$ in the center of infinite order. The restriction of the metric $d_{G}$ to the subgroup generated by $g$ defines a proper left invariant metric in $\mathbb{Z}=\langle g\rangle$. By Theorem 2.3 we know that two proper left invariant metrics defined in a group are coarsely equivalent; hence we can find an integer number $h_{1} \in \mathbb{Z}$ such that if $|h| \geq\left|h_{1}\right|$, then $\left\|g^{h}\right\|_{G} \geq a$. Let $p_{1}=1$, and for every $j=2 \ldots m$ we define $p_{j}$ as a sufficiently large number that satisfies $\sum_{i=1}^{j-1}(2 \cdot k \cdot m) 2^{p_{i}}<2^{p_{j}}$. Now take the finite set of integer numbers $\left\{h_{1}, \ldots, h_{m}\right\}$ with $h_{j}=2^{p_{j}} \cdot h_{1}$ for every $j=2, \ldots, m$. In this situation we create the norm $\|\cdot\|_{\omega}$ generated by the following system of weights (notice that the set of generators $S$ considered is the whole group):

$$
\omega(z)=\left\{\begin{array}{l}
\|z\|_{G} \text { if } z \neq g^{ \pm h_{i}} \text { for every } i=1 \ldots m, \\
C \text { otherwise. }
\end{array}\right.
$$

By the choice of $C$ and $\left\{h_{1}, \ldots, h_{m}\right\}$ it is clear that the first two conditions of the lemma are satisfied. To prove the third condition we define the homomorphism $f: \mathbb{Z}^{m} \rightarrow G$ as $f\left(x_{1}, \ldots, x_{m}\right)=g^{h}$ with $h=\sum_{i=1}^{m} x_{i} \cdot h_{i}$. Let us show that the restriction $\left.f\right|_{B(0, k)}: B(0, k) \rightarrow G$ to the ball of radius $k$ is a dilatation of constant $C$. It will be enough to check that

$$
\left\|g^{h}\right\|_{\omega}=\sum_{i=1}^{m}\left|x_{i}\right| \cdot C \text { if } h=\sum_{i=1}^{m} x_{i} \cdot h_{i} \text { and }\left|x_{i}\right| \leq k .
$$

The reasoning will be by contradiction. Suppose there exists an element of the form $g^{h}$ with $h=\sum_{i=1}^{m} x_{i} \cdot h_{i}$ and $\left|x_{i}\right| \leq k$ such that $\left\|g^{h}\right\|_{\omega}<\sum_{i=1}^{m}\left|x_{i}\right| \cdot C$. This implies that there exist an $r=\sum_{i=1}^{m} y_{i} \cdot h_{i}$ and an $s \in G$ such that $g^{h}=g^{r} \cdot s$ and

$$
\left\|g^{h}\right\|_{\omega}=\sum_{i=1}^{m}\left|y_{i}\right| \cdot C+\|s\|_{G}
$$

Notice that $\left|y_{i}\right| \leq k \cdot m$. There are now two possible cases:

Case 1: $s=1_{G}$. In this situation we have $\sum_{i=1}^{m}\left|y_{i}\right| \cdot C<\sum_{i=1}^{m}\left|x_{i}\right| \cdot C$, so there exists an $i$ such that $x_{i} \neq y_{i}$. Let $j=\max \left\{i \mid x_{i} \neq y_{i}\right\}$. From the fact $g^{h}=g^{r}$ we can deduce $\left(x_{j}-y_{j}\right) \cdot h_{j}=\sum_{i=1}^{j-1}\left(y_{i}-x_{i}\right) \cdot h_{i}$; it means $\left(x_{j}-y_{j}\right) \cdot 2^{p_{j}} \cdot h_{1}=$ $\sum_{i=1}^{j-1}\left(y_{i}-x_{i}\right) \cdot 2^{p_{i}} \cdot h_{1}$ and then

$$
2^{p_{j}} \leq\left|x_{j}-y_{j}\right| \cdot 2^{p_{j}} \leq \sum_{i=1}^{j-1}\left|y_{i}-x_{i}\right| \cdot 2^{p_{i}} \leq \sum_{i=1}^{j-1}\left|y_{i}\right|+\left|x_{i}\right| \cdot 2^{p_{i}} \leq \sum_{i=1}^{j-1} 2^{p_{i}} \cdot(k \cdot m+k)<2^{p_{j}},
$$

a contradiction. Therefore the first case is not possible.

Case 2: $s \neq 1_{G}$. In this case we have $g^{(h-r)}=s$ and $h-r \neq 0$ which implies $|h-r| \geq\left|h_{1}\right|$ and hence $\|s\|_{G} \geq a$. But from the fact that

$$
\left\|g^{h}\right\|_{\omega}=\sum_{i=1}^{m}\left|y_{i}\right| \cdot C+\|s\|_{G}<\sum_{i=1}^{m}\left|x_{i}\right| \cdot C
$$


we can deduce:

$$
a \leq\|s\|_{G}<\sum_{i=1}^{m}\left(\left|x_{i}\right|-\left|y_{i}\right|\right) \cdot C \leq \sum_{i=1}^{m}(k+k \cdot m) \cdot C \leq 2 \cdot k \cdot m^{2} \cdot C .
$$

Therefore $C \geq \frac{a}{2 \cdot k \cdot m^{2}}$, and this contradicts the choice of $a$ and $C$.

Here is the main theorem.

Theorem 4.2. If $G$ is a group such that its center is not locally finite, then there exists a proper left invariant metric $d_{G}$ such that $\operatorname{asdim}_{A N}\left(G, d_{G}\right)=\infty$.

Proof. We will use Corollary 3.4 and the previous lemma. Take any integer-valued proper left invariant metric $d$ in $G$ (see Remark 2.5) and some increasing sequences $\left\{k_{i}\right\}_{i \in \mathbb{N}}$ and $\left\{M_{i}\right\}_{i \in \mathbb{N}}$ of natural numbers. Let us construct the metric $d_{G}$ of the theorem by an inductive process.

Step 1: Apply the previous lemma to $d$ with $k=k_{1}, m=1$ and $R=M_{1}$. We obtain a proper left invariant metric $d_{\omega_{1}}$ such that the ball $B_{\omega_{1}}\left(1_{G}, R_{1}\right)$ is equal to the ball of radius $R_{1}$ of $d$. Also there exists a dilatation $f: B\left(0, k_{1}\right) \rightarrow G$ from the ball of radius $k_{1}$ of $\mathbb{Z}$ to $G$.

Induction Step: Suppose now that we have constructed a finite sequence of proper left invariant metrics $L=\left\{d_{\omega_{1}}, \ldots, d_{\omega_{n}}\right\}$ and a finite sequence of natural numbers $R_{1}<R_{2}<\ldots<R_{n}$ that satisfy the following conditions:

(1) $\|g\|_{\omega_{i}} \leq\|g\|_{\omega_{i-1}}$.

(2) $\|g\|_{\omega_{i}}=\|g\|_{\omega_{i-1}}$ if $\|g\|_{\omega_{i}} \leq R_{i}$.

(3) There exists a homomorphism $f_{i}: \mathbb{Z}^{i} \rightarrow G$ such that the restriction $\left.f_{i}\right|_{B\left(0, k_{i}\right)}$ is a dilatation in $\left(G, d_{\omega_{i}}\right)$ for every $i=1, \ldots, n$.

(4) $\operatorname{diam}\left(f_{i}\left(B\left(0, k_{i}\right)\right)\right)<R_{i+1}$ for every $i=1, \ldots, n-1$.

In these conditions define $R_{n+1}=\max \left\{M_{n+1}, R_{n}+1, \operatorname{diam}\left(f_{n}\left(B\left(0, k_{n}\right)\right)\right)\right\}$ and apply the previous lemma to $d_{\omega_{n}}$ with $k=k_{n+1}, m=n+1$ and $R=R_{n+1}$. We have now a new proper left invariant metric $d_{\omega_{n+1}}$. It is clear that the new finite sequence of proper left invariant metrics $\left\{d_{\omega_{n+1}}\right\} \cup L$ and the new finite sequence of numbers $R_{1}<R_{2}<\ldots<R_{n}<R_{n+1}$ satisfy the same four conditions.

Repeating this procedure we construct a sequence of integer-valued proper left invariant metrics $\left\{d_{\omega_{i}}\right\}_{i=1}^{\infty}$ and an increasing sequence of natural numbers $\left\{R_{i}\right\}_{i=1}^{\infty}$. By the first two properties and the fact that $\lim _{i \rightarrow \infty} R_{i}=\infty$ we deduce that for every $g \in G$ the sequence $\left\{\|g\|_{\omega_{i}}\right\}_{i=1}^{\infty}$ is asymptotically constant. Now define the function $\|\cdot\|_{G}: G \rightarrow \mathbb{N}$ by $\|g\|_{G}=\lim _{i \rightarrow \infty}\|g\|_{\omega_{i}}$. Again by the first two properties we can check that $\|\cdot\|_{G}$ is a proper norm, so it defines a proper left invariant metric $d_{G}$. Using the third and fourth properties we have that for every $i \in \mathbb{N}$ there exists a homomorphism $f_{i}: \mathbb{Z}^{i} \rightarrow G$ such that the restriction to the ball $B\left(0, k_{i}\right)$ is a dilatation in $\left(G, d_{G}\right)$. Because in each step we are increasing the dimension of the balls, we get that for every $m \in \mathbb{N}$ the metric space $\left(G, d_{G}\right)$ satisfies the conditions of Corollary 3.4, so we get $\operatorname{asdim}_{A N}\left(G, d_{G}\right) \geq m$. Therefore $\operatorname{asdim}_{A N}\left(G, d_{G}\right)=\infty$.

Recall that in 11] Dranishnikov and Smith showed that the asymptotic dimension of finitely generated nilpotent groups is equal to the Hirsch length of the group. Hence the asymptotic dimension of a nilpotent group is always finite for every proper left invariant metric. The next corollary shows that the unique nilpotent 
groups that satisfy the same property for the asymptotic Assouad-Nagata dimension are the finite ones.

Corollary 4.3. Let $G \neq 1$ be a finitely generated nilpotent group. $G$ is finite if and only if $\operatorname{asdim}_{A N}\left(G, d_{G}\right)=0$ for every proper left invariant metric $d_{G}$.

Proof. For one implication just use that the asymptotic Assouad-Nagata dimension is zero for all bounded spaces. For the converse we can apply the main theorem and the well known fact that says that if the center of a locally finite group is locally finite, then the whole group is finite.

Remark 4.4. We were informed that M. Zarichnyi recently solved the problems of Dranishnikov for the case $G=\mathbb{Z}$ only.

\section{REFERENCES}

[1] P. Assouad, Sur la distance de Nagata, C. R. Acad. Sci. Paris Sér. I Math. 294 (1982), no. 1, 31-34. MR651069 (83b:54034)

[2] G. Bell, A. Dranishnikov, On asymptotic dimension of groups acting on trees, Geom. Dedicata 103 (2004), 89-101. MR2034954 (2005b:20078)

[3] G. Bell, A. N. Dranishnikov, J. E. Keesling, On a formula for the asymptotic dimension of free products, Fund. Math. 183 (2004), no. 1, 39-45. MR2098148 (2005g:20064)

[4] G. Bell, A. Dranishnikov, A Hurewicz-type theorem for asymptotic dimension and applications to geometric group theory, Trans. Amer. Math. Soc. 358 (2006), no. 11, 4749-4764. MR2231870 (2007e:20092)

[5] G. Bell, A. Dranishnikov, Asymptotic dimension, Topology and its Appl. 155 (2008), no. 12, $1265-1296$.

[6] N. Brodskiy, J. Dydak, J. Higes, A. Mitra, Dimension zero at all scales, Topology and its Appl. 154 (2007), no. 14, 2729-2740. MR2340955 (2008k:54049)

[7] N. Brodskiy, J. Dydak, J. Higes, A. Mitra, Nagata-Assouad dimension via Lipschitz extension, Israel J. Math. 171 (2009), 405-423.

[8] N. Brodskiy, J. Dydak, M. Levin, A. Mitra, Hurewicz theorem for Assouad-Nagata dimension, J. Lond. Math. Soc. (2) 177 (2008), no. 3, 741-756.

[9] N. Brodskiy, J. Dydak, U. Lang, Assouad-Nagata dimension of wreath products of groups, preprint, math.MG/0611331v2.

[10] A. Dranishnikov, Open problems in asymptotic dimension theory, preprint, available at http://aimbri12.securesites.net/pggt.

[11] A. Dranishnikov, J. Smith, Asymptotic dimension of discrete groups, Fund. Math. 189 (2006), no. 1, 27-34. MR2213160 (2007h:20041)

[12] A. Dranishnikov, J. Smith, On asymptotic Assouad-Nagata dimension, Topology and its Appl. 154 (2007), no. 4, 934-952. MR2294641 (2008m:54041)

[13] A. Dranishnikov, M. Zarichnyi, Universal spaces for asymptotic dimension, Topology and its Appl. 140 (2004), no. 2-3, 203-225. MR2074917(2005e:54032)

[14] C. Drutu, Quasi-isometry invariants and asymptotic cones, Int. J. Algebra Comput. 12 (2002), no. 1-2, 99-135. MR1902363 (2003g:20069)

[15] J. Dydak, J. Higes, Asymptotic cones and Assouad-Nagata dimension, Proc. Amer. Math. Soc. 136 (2008), 2225-2233. MR 2383529(2009j:54051)

[16] M. Gromov, Asymptotic invariants for infinite groups, in Geometric Group Theory, vol. 2, 1-295, G. Niblo and M. Roller, eds., Cambridge University Press, 1993. MR.1253544 (95m:20041)

[17] M. Gromov, Groups of polynomial growth and expanding maps, Inst. Hautes Études Sci. Publ. Math. (1981), no. 53, 53-73. MR623534 (83b:53041)

[18] J. Higes, Assouad-Nagata dimension of locally finite groups and asymptotic cones, preprint, math.MG0711.1512.

[19] U. Lang, T. Schlichenmaier, Nagata dimension, quasisymmetric embeddings, and Lipschitz extensions, IMRN International Mathematics Research Notices (2005), no. 58, 3625-3655. MR:2200122(2006m:53061) 
[20] P.W. Nowak, On exactness and isoperimetric profiles of discrete groups, Journal of Functional Analysis 243 (2007), no. 1, 323-344. MR2291440 (2008c:20081)

[21] D. Osin, Asymptotic dimension of relatively hyperbolic groups, Int. Math. Res. Not. (2005), no. 35, 2143-2161. MR2181790 (2006g:20068)

[22] J. Roe, Hyperbolic groups have finite asymptotic dimension, Proc. Amer. Math. Soc. 133 (2005), no. 9, 2489-2490. MR2146189 (2005m:20102)

[23] Y. Shalom, Harmonic analysis, cohomology, and the large-scale geometry of amenable groups, Acta Math. 192 (2004), 119-185. MR2096453 (2005m:20095)

[24] J. Smith, On asymptotic dimension of countable abelian groups, Topology Appl. 153 (2006), no. 12, 2047-2054. MR2237596 (2007g:20044)

[25] J. Smith, The asymptotic dimension of the first Grigorchuk group is infinity, Rev. Mat. Complut. 20 (2007), no. 1, 119-121. MR2310581 (2007k:20095)

[26] K. Whyte, Amenability, bi-Lipschitz equivalence, and the von Neumann conjecture, Duke Journal of Mathematics 99 (1999), 93-112. MR1700742 (2001a:20064)

Departamento de Geometría y Topología, Facultad de Ciencias Matemáticas, UniVersidad Complutense de Madrid, Madrid, 28040, Spain

E-mail address: josemhiges@yahoo.es 